\title{
Tag Separation in Cardiac Tagged MRI
}

\author{
Junzhou Huang ${ }^{1}$, Zhen Qian ${ }^{1}$, Xiaolei Huang ${ }^{2}$, Dimitris Metaxas ${ }^{1}$, \\ and Leon $\mathrm{Axel}^{3}$
}

${ }^{1}$ Division of Computer and Information Sciences, Rutgers University, NJ, USA

${ }^{2}$ Department of Computer Science and Engineering, Lehigh University, PA, USA

${ }^{3}$ Department of Radiology, New York University, New York, USA

\begin{abstract}
In this paper we introduce a tag separation method for better cardiac boundary segmentation and tag tracking. Our approach is based on two observations in the cardiac tagged MR images: 1) the tag patterns have a regular texture; 2) the cardiac images without tag patterns are piecewise smooth with sparse gradients. These observations motivate us to use two dictionaries, one based on the Discrete Cosine Transform for representing tag patterns and the other based on the Wavelet Transform for representing the underlying cardiac image without tag patterns. The two dictionaries are built such that they can lead to sparse representations of the tag patterns and of the piece-wise smooth regions without tag patterns. With the two dictionaries, a new tag separation approach is proposed to simultaneously optimize w.r.t. the two sparse representations, where optimization is directed by the Total Variation regularization scheme. While previous methods have focused on tag removal, our approach to acquiring both optimally-decomposed tag-only image and the cardiac image without tags simultaneously can be used for better tag tracking and cardiac boundary segmentation. We demonstrate the superior performance of the proposed approach through extensive experiments on large sets of cardiac tagged MR images.
\end{abstract}

\section{Introduction}

The tagged MRI technique generates a set of tag patterns within the myocardium as temporary markers at end-diastole by spatial modulation of the magnetization. These tagged patterns appear initially in darker colors in the tagged MR images. Generally, they will persist for a short period of time in the myocardium and deform with the underlying tissue during the cardiac cycle in vivo, which provides detailed myocardial motion information. Sample images are shown in Figure 1. Although the added tagged patterns can facilitate the visualization of detailed transmural motion, they also pose great challenges to the cardiac image processing and analysis tasks, such as cardiac boundary segmentation.

To address this problem, several approaches have been proposed to remove the tag patterns before performing segmentation. In [1] 2], morphological operations are used to fill in the region between removed tagging lines. However, it is difficult to choose reasonable parameters for a large set of tagged MR images. In [3] [4, band-pass filtering approaches are proposed to enhance the tag-patterned region

D. Metaxas et al. (Eds.): MICCAI 2008, Part II, LNCS 5242, pp. 289 297, 2008.

(C) Springer-Verlag Berlin Heidelberg 2008 


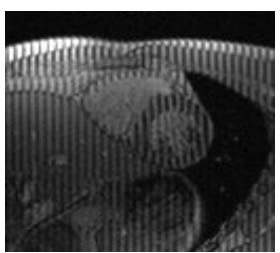

(a)

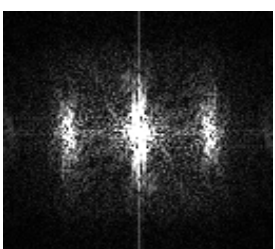

(b)

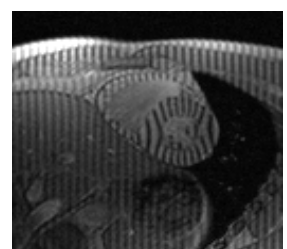

(c)

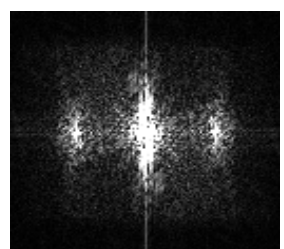

(d)

Fig. 1. Tagged cardiac MR images and their corresponding magnitude spectrums

and increase the blood-to-myocardium contrast. However, the above filteringbased approaches greatly depend on the designed filters. They often do not adapt well to large sets of images captured under different conditions. To avoid these problems, a band-stop filtering based method was recently proposed for tag removal [5. The images are processed in the spectral domain. Based on observations from large sets of MR images with tagging lines, they found that, except for the low frequency peak at the origin, other harmonic energy peaks placed symmetrically around the low frequency peak may be introduced by the spatial tag patterns (as shown in Figure1 (b, d)). According to this observation, the optimal band-stop filter based on the Mean Shift algorithm is designed to remove these harmonic energy peaks while keeping other frequency components unchanged as much as possible. Experimental results on 150 cardiac images with tagging lines demonstrate the superior performance over previous approaches.

However, this method still has drawbacks: 1) although it can localize the harmonic frequency regions which correspond to the spatial tagging lines, it abandons all frequency information in the localized regions, which include not only the tagging-line related frequency information but also those related to the piecewise smooth images without tagging lines. Thus, it inevitably causes some artifacts in the recovered images after tag removal; 2) when there are only a few harmonic regions, these artifacts will not be obvious since they only remove relatively less distinctive information from piecewise smooth images. However, the artifacts will be more serious when there are more harmonic regions corresponding to the spatial tag patterns in the tagged images. Thus, simply removing all frequency information in these regions will rapidly degrade the tag removal performance; 3) as all previous methods, it does not show the potential for better tag tracking, due to the lack of recovering the tag-only image.

In this paper we develop a tag separation approach according to the image decomposition theory based on sparse representations. We observe that the tag pattern appearance in the cardiac tagged MR images is approximately a regular texture, and that without the tag patterns, the cardiac images are piecewise smooth with sparse gradients. These observations motive us to seek sparse representations for both the tag patterns and the underlying piece-wise smooth image region. Two dictionaries are found appropriate. One is based on the Discrete Cosine Transform (DCT) for representing the regular tag-pattern texture, 
and the other is based on the Wavelet Transform (WT) for representing the image without tags. Using these two dictionaries, the tag patterns are separated from the original tagged images by jointly optimizing over the separated tag patterns and the remaining piecewise smooth regions, under the supervision of the Total Variation regularization scheme.

Different from previous tag removal methods, our tag separation approach can optimally decompose the tagged image into one tag-only image and one remaining piecewise-smooth cardiac image. The tag-only image can then be used for tag tracking, and the remaining piecewise smooth image without tag patterns can be used for accurately localizing the cardiac boundaries. We demonstrate the superior performance of the proposed method through extensive experiments on large sets of cardiac tagged MR images, and show that the method outperforms previous methods.

The remainder of the paper is organized as follows. Section 2 introduces the problem formulation. The proposed approach is detailed in section 3. Experimental results and discussions are presented in section 4, and we conclude this paper in section 5 .

\section{Problem Formulation}

If we know the tag patterns exactly in the tagged cardiac MR images, the detailed myocardial motion information can be obtained by tracking these known tag patterns in the image sequences. If we know the piecewise smooth cardiac images without tag patterns, we can easily segment the cardiac boundary by known techniques, such as the Metamorphs model 6]. However, we only know their combination when given the tagged cardiac MR images. Therefore, it is important to decompose a tagged MR image into two separate parts: the tags, and the piecewise smooth image without the tags.

We assume that a known tagged cardiac MR image $I$ can be represented as the combination of a piecewise smooth cardiac image $S$ without tags and the tag-only image $T$ :

$$
I=S+T
$$

where the images $I, S$ and $T$ are of the same size $n \times n$. They are then reordered as $1 \mathrm{D}$ vectors with size $n^{2} \times 1$. The decomposition problem is a severely under-constrained problem. It is impossible to obtain good solutions for $S$ and $T$ only given $I$ without prior information. Fortunately, two priors are observed from large sets of tagged cardiac MR images: 1) the tag patterns appear as a kind of regular texture in the tagged cardiac MR images; 2) the cardiac images without tag patterns are piecewise smooth with sparse gradients. Previous work has established that the piecewise smooth images can be sparsely represented by the undecimated version of the Wavelet Transforms (UWT) while the DCT is appropriate for sparsely representing the textures [7.Thus, the above observed priors motivate us to build two appropriate over-complete dictionaries $A \in \mathbb{R}^{n^{2} \times m}$ and $B \in \mathbb{R}^{n^{2} \times m}$, where typically $m \gg n^{2}$. While the dictionary $A$ 
is based on the DCT for representing tag patterns, the dictionary $B$ is based on the UWT for representing the cardiac image without tag patterns. Dictionaries $A$ and $B$ are built such that they can lead to sparse representations over either the tag texture or the piecewise smooth image.

Therefore, the tag separation problem is to optimally decompose image $I$ into two images $S$ and $T$, where the image $S$ should be sparsely represented by dictionary $B$ and the tag image $T$ should be sparsely represented by dictionary $A$. The problem is formulated as the image decomposition problem:

$$
(\hat{\alpha}, \hat{\beta})=\underbrace{\operatorname{argmin}}_{\alpha, \beta}\left(\|\alpha\|_{0}+\|\beta\|_{0}\right), \text { while } I=A * \alpha+B * \beta
$$

where $\alpha$ and $\beta$ are sparse vectors with size $m \times 1$.

\section{Algorithm}

Although the tag separation was formulated as an image decomposition problem in Eq. 2, the problem in Eq. 2 is NP-hard. In the general case, no known procedure can correctly find the sparsest solution more efficiently than exhausting all subsets of the entries for $\alpha$ and $\beta[8$. Fortunately, recent developments in sparse representation provide a theorem to efficiently solve this problem [7:

Definition. Given a matrix $Q$, its Mutual Incoherence $M_{Q}$ is defined as the maximal off-diagonal entry in the absolute Gram matrix $\left|Q^{H} * Q\right|$.

Theorem. If the image $I$ is the combination of image $T$ and image $S, T=A * \alpha$, $S=B * \beta, Q=[A, B]$, and $\|\hat{\alpha}\|_{0}+\|\hat{\beta}\|_{0}<0.5 *\left(1+1 / M_{Q}\right)$ is true, then the solution to Eq. 3 leads to the global optimal solution of the $l^{0}$ minization problem in Eq. 2, and this is the desired image decomposition.

This Theorem implies that, if the true $\alpha$ and $\beta$ are sparse enough, the image decomposition problem in Eq. 2 is equivalent to the following $l^{1}$ minimization problem:

$$
(\hat{\alpha}, \hat{\beta})=\underbrace{\operatorname{argmin}}_{\alpha, \beta}\left(\|\alpha\|_{1}+\|\beta\|_{1}\right), \text { while } I=A * \alpha+B * \beta
$$

It is a convex optimization problem and can be easily solved. Thus, according to the above theorem, if the input image is exactly combined from one piecewise smooth image and another tag pattern image, we definitely can successfully decompose it into the two desired output images. Unfortunately, in practice, the input tagged cardiac MR images inevitably include noise. It is impractical that the input images are exactly combined from a tag pattern and a piecewise smooth image. Following the idea in [7, we approximate this problem by relaxing the constraint in Eq. 3 for denoising and we add the Total Variation (TV) term for an additional penalty:

$$
(\hat{\alpha}, \hat{\beta})=\underbrace{\operatorname{argmin}}_{\alpha, \beta}\left(\|\alpha\|_{1}+\|\beta\|_{1}+\lambda *\|I-A * \alpha-B * \beta\|^{2}+\gamma * T V(B * \beta)\right)(4)
$$


Here, the TV penalty term can guide the image $S$ to fit the piecewise smooth model based on the assumption that the cardiac image without tag patterns should be a piecewise smooth image with sparse gradients. This formulation can accelerate the convergence. We know that $T=A * \alpha$ and $S=B * \beta$. When they are put back into Eq. 4, it is further approximated as follows:

$$
(\hat{T}, \hat{S})=\underbrace{\operatorname{argmin}}_{T, S}\left(\left\|A^{+} * T\right\|_{1}+\left\|B^{+} * S\right\|_{1}+\lambda *\|I-T-S\|^{2}+\gamma * T V(S)\right)(5)
$$

where $A^{+}$and $B^{+}$are Moore-Penrose pseudo inverses of $A$ and $B$ respectively. Their computation is relatively easy since $A$ and $B$ are two dictionaries corresponding to the DCT and the UWT, respectively. It is known that there exist fast algorithms for DCT and UWT with complexity of no more than $n^{2} \log _{2}(n)$ while the image size is $n \times n$. The optimization to find the solution in Eq. 5 can be efficiently performed by the Block-coordinate Relaxation algorithm 9 .

Now, the tagged cardiac image $I$ can be efficiently and optimally decomposed into the tag pattern only image $T$ and the piecewise smooth cardiac image $S$. Then, the cardiac boundary segmentation can be performed on the piecewise smooth cardiac image $S$ instead of the tagged cardiac image $I$, and the tag tracking can be performed on the tag pattern only image $T$ instead of $I$. The experimental results in the next section will demonstrate that our tag separation approach can facilitate both tasks of cardiac boundary segmentation and tag tracking and leads to more accurate results.

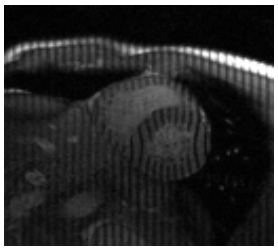

(a)

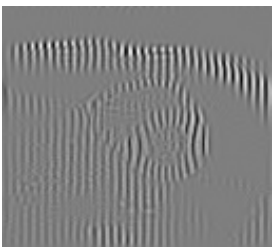

(b)

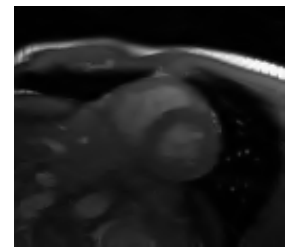

(c)

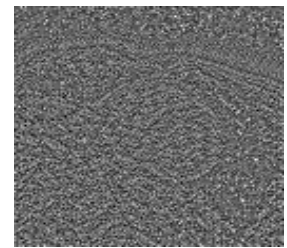

(d)

Fig. 2. Tag separation. (a) the input image $I$; (b) the separated tag $T$; (c) the piecewise smooth image $S$; (d) noise.

\section{Experiments}

The testing data are 4D spatial-temporal short-axis cardiac tagged MR images. A 1.5T Siemens MR imaging system was used to acquire the images, using an ECG-gated tagged gradient echo pulse sequence. These images are perpendicular to an axis through the center of the LV. Each set consists of $18-24$ phases, with $5-7$ slices (images) per phase. We collected 60 sequences and 1200 2D-images. An expert was asked to draw the endocardium contours and the tagged lines in these images for validation purposes.

In the following, we demonstrate the superior performance of the proposed tag separation approach for better cardiac boundary segmentation and tag tracking 
on the above tested data. Our algorithm was implemented in MATLAB and tested on a $2 \mathrm{GHz}$ Pentium $4 \mathrm{PC}$. The average running time was 8 seconds for an image with size $128 \times 128$. Figure 2 shows one example of our tag separation approach. Thanks to the efficient image decomposition based on sparse representation, our approach almost perfectly decomposes the input tagged image $I$ into the tag image $T$, the piecewise smooth cardiac image $S$, and noise.

To compare the band-pass filtering method [4, band-stop filtering method 5] and the proposed approach, we used them on the same set of testing data. Figure 3 (1) shows the comparisons on the whole image in terms of visual effect. Figure 3 (2) and (3) show the comparisons on local regions of the image. It shows that the results by the band-stop filtering method obtain better visual effects than those by band-pass filtering method. It is consistent with that presented in [5]: the band-stop filtering method is better than the band-pass filtering method for tag removal. With further observations in Figure 3 (1c), we can find that there are some artifacts in the recovered images by the band-stop filtering method. These artifacts are more when zooming in to focus on only the local regions, as shown in Figure 3 (2c) and (3c). This confirms a limitation of the band-stop filtering method: it can easily cause artifacts in the recovered images, since it simply abandons all frequency information in the localized harmonic frequency regions. Thanks to the efficient joint optimization and denoising schemes, our approach can recover clear results as shown in Figure 3 (d).

Note that the purpose of the tag separation is to obtain more accurate cardiac boundary segmentation and better tag tracking. Therefore, we applied the Metamorphs model [6] to segment the LV endocardium on the cardiac images without tag patterns, which are recovered by different methods. Figure [3 (4) shows one segmentation example. It is obvious that the estimated LV endocardium contours by our approach are closer to the true boundary than those obtained by the other two methods, while the results by the band-stop filtering method are better than those obtained by the band-pass filtering method. Quantitative validation is performed by comparing the segmentation results with the expert solution. The above experimental results show that our approach and the band-stop filtering method are far better than the band-pass filtering method 4 for tag removal. We therefore only focus on the quantitative comparison between our approach and the band-stop filtering method [5]. Denote the expert segmentation in the images as $l_{\text {true }}$, and the results from the recovered images as $l_{\text {est }}$. We define the False Positive Fraction (FPF) to indicate the amount of tissue falsely identified by the estimation as a fraction of the total amount of tissue in the true segmentation: $\frac{\left|l_{\text {est }}-l_{\text {true }}\right|}{l_{\text {true }}}$. The Positive Fraction (TPF) describes the fraction of the total amount of tissue in the true segmentation that is overlapped with the estimation: $\frac{\left|l_{\text {est }} \cap l_{\text {true }}\right|}{l_{\text {true }}}$. The quantitative comparison result is: the band-stop filtering method gained $F P F=19.6 \%$ and $T P F=83.8 \%$, while our approach achieved $F P F=9.3 \%$ and $T P F=91.7 \%$. The proposed approach thus outperforms the band-stop filtering method in endocardium segmentation.

To our knowledge, our method is the first to optimally separate the tag patterns for tracking using image decomposition and sparse representations. For 
(1)
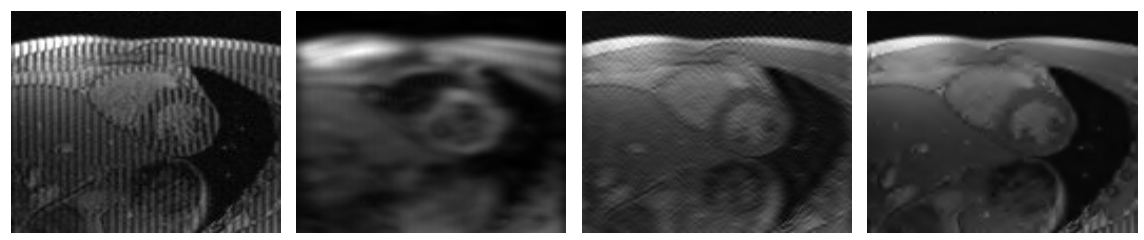

(2)
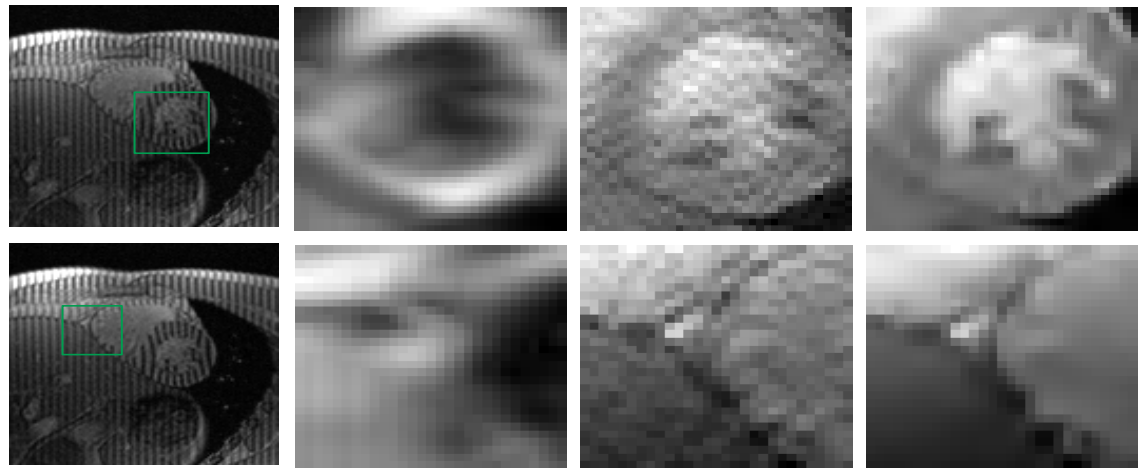

(4)

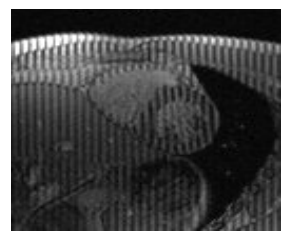

(a)

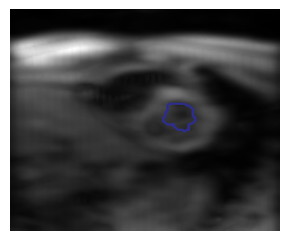

(b)

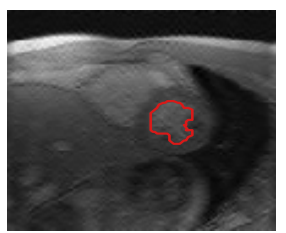

(c)

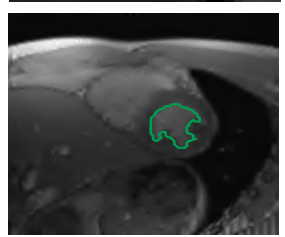

(d)

Fig. 3. Comparisons. (1)visual comparisons in the whole images; (2) and (3) visual comparisons in the local regions; (4) segmentation comparisons; (a) tagged images; (b) the band-pass method [4; (c) the band-stop method [5]; (d) the proposed approach.

comparisons, we use a set of snakes to track the tags in the original tagged image $I$ and the separated tag only image $T$ respectively. Figure 4 (a) and (c) show the tracking results in the original tagged images. Some mis-tracks occur in the regions indicated by green circles. Figure 4 (b) and (d) show the corresponding tracking results in the separated tag only images by our approach. The new results based on $T$ by the proposed approach are better than those obtained on the original image $I$. We further test the average and maximum tracking error in the different periods, such as Middle of Systole (MS), End of Systole (ES), Middle of Diastole (MD) and End of Diastole (ED). Table 1 tabulates the comparison on one example tagged image sequence. Quantitative comparisons of tracking results are also conducted on all cardiac image sequences. Denote tracked motions by the expert as $m_{\text {true }}$, and the tracked results by snakes as $m_{\text {track }}$. We define the Correct Tracked Rate (CTR) of the motions as: $\frac{\left|m_{\text {track }}-m_{\text {true }}\right|}{\left|m_{\text {true }}\right|}$. While the CTR on all testing sequences is $72.8 \%$, the CTR on all corresponding tag-only sequences (separated by our approach) is $92.3 \%$. The above experimental results show that our approach can facilitate better tag tracking. 


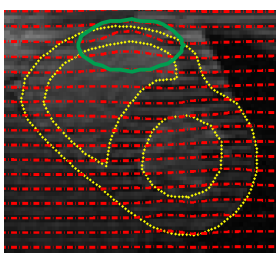

(a)

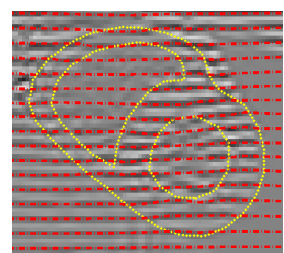

(b)

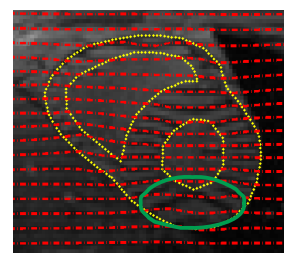

(c)

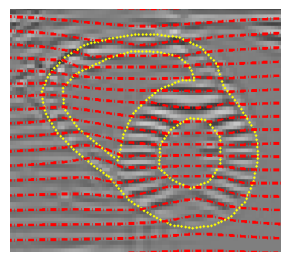

(d)

Fig. 4. Tag tracking comparisons. (a) and (c)mis-track occurs in the areas indicated by the green circles in the original tagged image sequence; (b) and (d) the corresponding results by our approach.

Table 1. The average/maximum tracking error comparison (pixels)

\begin{tabular}{|l||c|c|c|c|}
\hline Tracking error (pixel) & MS & ES & MD & ED \\
\hline Old (Avg/Max) & $0.0897 / 4.2734$ & $0.1880 / 4.3155$ & $0.2311 / 3.9458$ & $0.1497 / 3.9586$ \\
\hline Proposed (Avg/Max) & $0.0302 / 0.9387$ & $0.0378 / 0.9780$ & $0.0397 / 0.9629$ & $0.0362 / 0.9623$ \\
\hline
\end{tabular}

\section{Conclusion}

We have presented a tag separation approach for tagged cardiac MR images. Our approach has the following contributions. First, we introduce one self-adaptive image decomposition method for tag separation, which can be easily extended to other medical image applications. Second, the joint optimization framework plus denoising makes our approach very effective for tag separation task. Third, it is unique that our approach can optimally recover not only the cardiac image without tag patterns for accurate cardiac boundary segmentation, but also the tag only images for better tag tracking. The potential of our approach is demonstrated through extensive experiments using large sets of tagged cardiac MR images. Our method is also shown to outperform several previous methods on tag separation, boundary segmentation and tag tracking.

\section{References}

1. Guttman, M., Prince, J., McVeigh, E.: Tag and contour detection in tagged MR images of the left ventricle. IEEE Trans. on Medical Imaging 13(1), 74-88 (1994)

2. Montillo, A., Metaxas, D., Axel, L.: Automated segmentation of the left and right ventricles in $4 \mathrm{~d}$ cardiac spamm images. In: Medical Imaging Computing and Computer-Assisted Intervention, pp. 620-633 (2002)

3. Osman, N., Kerwin, W., McVeigh, E., Prince, J.: Cardiac motion tracking using CINE harmonic phase HARP magnetic resonance imaging. Magnetic Resonace in Medicine 42, 1048-1060 (1999)

4. Manglik, T., Axel, L., Qian, Z., Pai, W., Kim, D., Dugal, P., MontilloJones, A., Metaxas, D.: Use of bandpass gabor filters for enhancing blood-myocardium contrast and filling-in tags in tagged MR images. In: International Society for Magnetic Resonance In Medicine, vol. 1193 (2004) 
5. Qian, Z., Huang, R., Metaxas, D., Axel, L.: A novel tag reoval technique for tagged cardiac mri and its applications. In: IEEE International Symposium on Biomedical Imaging: From Nano to Macro., pp. 364-367 (2007)

6. Huang, X., Metaxas, D., Chen, T.: Metamorphs: Deformable shape and texture models. In: IEEE Conf. on Computer Vision and Pattern Recognition, pp. 496-503 (2004)

7. Starck, J., Elad, M., Donoho, D.: Image decomposition via the combination of sparse representations and a variational approach. IEEE Trans. on Image Processing 14(10), 1570-1582 (2005)

8. Candes, E., Tao, T.: Near-optimal signal recovery from random projections: Universal encoding strategies. IEEE Transactions on Information Theory 52(12), 54065425 (2006)

9. Bruce, A., Tseng, S.S., Block, P.: coordinate relaxation methods for nonparametric signal de-noising. In: Proceedings of SPIE, vol. 3391, pp. 75-86 (1998) 